\title{
Optimizing compliance with surgical antimicrobial prophylaxis guidelines in patients undergoing gastrointestinal surgery at a referral teaching hospital in southern Iran: clinical and economic impact
}

This article was published in the following Dove Press journal: Infection and Drug Resistance

\section{Laleh Mahmoudi \\ Mehrdad Ghouchani' \\ Motahareh Mahi-Birjand ${ }^{2}$ \\ Alimohammad Bananzadeh ${ }^{3}$ \\ Ali Akbari ${ }^{4}$ \\ 'Department of Clinical Pharmacy, School of Pharmacy, Shiraz University of Medical Sciences, Shiraz, Iran; ${ }^{2}$ Infectious Disease Research Center, Birjand University of Medical Sciences, Birjand, Iran; ${ }^{3}$ Colorectal Research Center, Shiraz University of Medical Sciences, Shiraz, Iran; ${ }^{4}$ Department of Anesthesiology, Medical School, Shiraz University of Medical Sciences, Shiraz, Iran}

Correspondence: Ali Akbari

Department of Anesthesiology, Medical

School, Shiraz University of Medical

Sciences, Shiraz, Iran

Tel/Fax +987132125400

Email akbaria@sums.ac.ir
Background: Antibiotic prophylaxis is one of the major approaches to prevent surgical site infection. Despite the availability of international guidelines on it, the practice of antibiotic prophylaxis is still far from optimal. This study aimed to assess the impact of guideline implementation on the rational use of prophylactic antibiotics and its cost-saving effect in gastrointestinal surgery by clinical pharmacist intervention.

Methods: A pre and post intervention study was carried out between October 2017 and June 2018 on patients who underwent gastrointestinal surgery in a major referral teaching hospital in Shiraz, southern Iran. The intervention phase consisted of revising the institutional guidelines for surgical antimicrobial prophylaxis, assigning a clinical pharmacist to the surgical department, and arranging several meetings with the surgical department regarding the appropriate utilization of antibiotics. Differences in antibiotic utilization in patients before and after the intervention phase were compared. Exposures were surgical antimicrobial prophylaxis timing and agents, and main outcomes were incidence of surgical site infection and length of hospital stay.

Results: A total of 430 patients were included in the post intervention phase, while 445 patients were included in the baseline evaluation. Promoting appropriate antibiotic use in the post intervention group had the potential to decrease medication costs by reducing unnecessary prescriptions and duration of antibiotic usage $(P<0.001)$. In the pre intervention group, the mean cost of antibiotic prescriptions was 11.5 times that of the post intervention group. Mean hospitalization in the pre intervention group was greater than the post intervention group $(P<0.001)$. Furthermore, our data revealed that the rate of postsurgery infection in the post intervention group was $3.03 \%$, while this rate was $6.76 \%$ in the preintervention group $(P=0.01)$. Conclusion: This study provides evidence that adherence to surgical antimicrobial prophylaxis guidelines increased the rational use of prophylactic antibiotics, with substantial cost savings in patients who underwent surgery.

Keywords: prophylaxis antibiotic, efficacy of guideline, IDSA guideline, cost saving

\section{Plain language summary}

Surgical site infection is the leading cause of unplanned and potentially preventable hospital readmission in surgical patients. Prescribing antibiotics before surgery is a well-proved approach to reduce the infection risk in patients underwent surgery. But, overuse of these 
drugs is observed in many hospitals of developing countries which increases the risk of drug resistance and imposes financial burden on patients and hospitals. Therefore, implementation of standard protocols can overcome these challenges. In this study, we designed a program including revising the institutional protocol, assigning a clinical pharmacist to the surgical department, and arranging several meetings with surgical department regarding the appropriate utilization of antibiotics. We surveyed different parameters before and after the designed program such as incidence and rate of infection, choice of prophylactic drugs, duration of drugs use, length of hospital stay, and financial costs. We found that the quality improvement project and implementation of protocol significantly improved rational use of antibiotics among gastrointestinal surgeons and residents as well as reduced drug usage costs and length of hospital stay.

\section{Introduction}

Surgical site infection (SSI) is the leading cause of unplanned and potentially preventable hospital readmission in surgical patients. ${ }^{1,2}$ It is considerably involved in surgery-related morbidity and increased health-care costs. ${ }^{3}$ Depending on the operative site and degree of contamination, SSIs occur in up to $10 \%$ of surgeries. ${ }^{4}$ However, this rate can vary between populations. For instance, it has been reported that there is an adverse relationship between the SSI rate and Human Development Index (HDI). The SSI rate in high-HDI countries is $9.4 \%$, while these rates in the middle-HDI and low-HDI countries are $14.0 \%$ and $23.2 \%$, respectively. ${ }^{5}$ According to a report, the incidence of health care-associated infections (HAIs) in Iran is 1.18, with $14.6 \%$ SSIs. ${ }^{6}$

The Iranian Center for Communicable Disease Control is responsible for monitoring HAIs in Iran. ${ }^{7}$ As mentioned, SSIs comprise a remarkable share of HAIs in Iran. ${ }^{6}$ Because of the devastating complications of SSIs, a great number of attempts have been made to prevent or reduce this surgical complication. Surgical antibiotic prophylaxis (SAP) has become an accepted part of surgical practice to prevent infections at the surgical site and optimize postoperative recovery. ${ }^{8}$ The efficiency of AP in inhibiting operative infections has been confirmed in numerous investigations. It has been well proven that infection incidence in surgical patients with no AP is significantly higher than those receiving prophylactic antibiotics. ${ }^{9,10}$

The guidelines were introduced to provide practitioners with a standardized approach to the safe and effective use of antibiotic drugs for the prevention of SSIs, based on currently available clinical indications and emerging issues. ${ }^{9}$ During the last few decades, several papers have been published on optimal AP, and in this regard international guidelines for surgical prophylaxis have been updated and propagated. ${ }^{11}$ Despite this, studies have shown that optimal practice is not achieved in many hospitals, especially in developing countries. ${ }^{12,13}$ The knowledge and attitudes of surgeons about appropriate SAP are important factors in the implementation of guidelines. It is necessary for hospital administrators and institutional quality-improvement departments to provide approaches to reduce such outcomes of antibiotic overuse as increased antibiotic resistance and higher financial costs to hospitals. To address this gap, we designed this study to determine the efficacy of adherence to SAP guidelines clinically and economically.

\section{Methods}

\section{Setting and data collection}

Patients admitted to the gastrointestinal surgical ward of Shahid Faghihi Hospital of Shiraz University, which is a high-volume tertiary teaching hospital, were included in this study. This was a prospective cross-sectional study conducted from October 2017 to June 2018 (Effective Practice and Organisation of Care taxonomy: implementation strategies [category]/authority and accountability for quality of practice [subcategory]) performed in two phases: phase 1 between October 2017 and January 2017 (pre intervention group) and phase 2 between March 2018 and June 2018 (postintervention group). Patients were included if they had undergone gastrointestinal surgery (Table 1). Exclusion criteria were patients who had received therapeutic antibiotics to treat bacterial infections prior to the intervention procedure or who had symptoms of infection after the intervention procedure and if they declined to provide informed consent. The study was in accordance with the Declaration of Helsinki (1975) and local regulations. It was also approved by the ethical committee of the Shiraz University of Medical Sciences (94-01-05-10183). Written consent was obtained from all study participants or their legal guardians.

\section{Study description}

The criteria of prophylactic antibiotic-usage evaluation were established based on the guidelines developed jointly by the American Society of Health-System Pharmacists, Infectious Diseases Society of America (IDSA), Surgical Infection Society, and Society for Healthcare Epidemiology of America (Table 1). ${ }^{14}$ 
Table I Summary of Infectious Diseases Society of America guideline recommendations for antimicrobial prophylaxis in adults

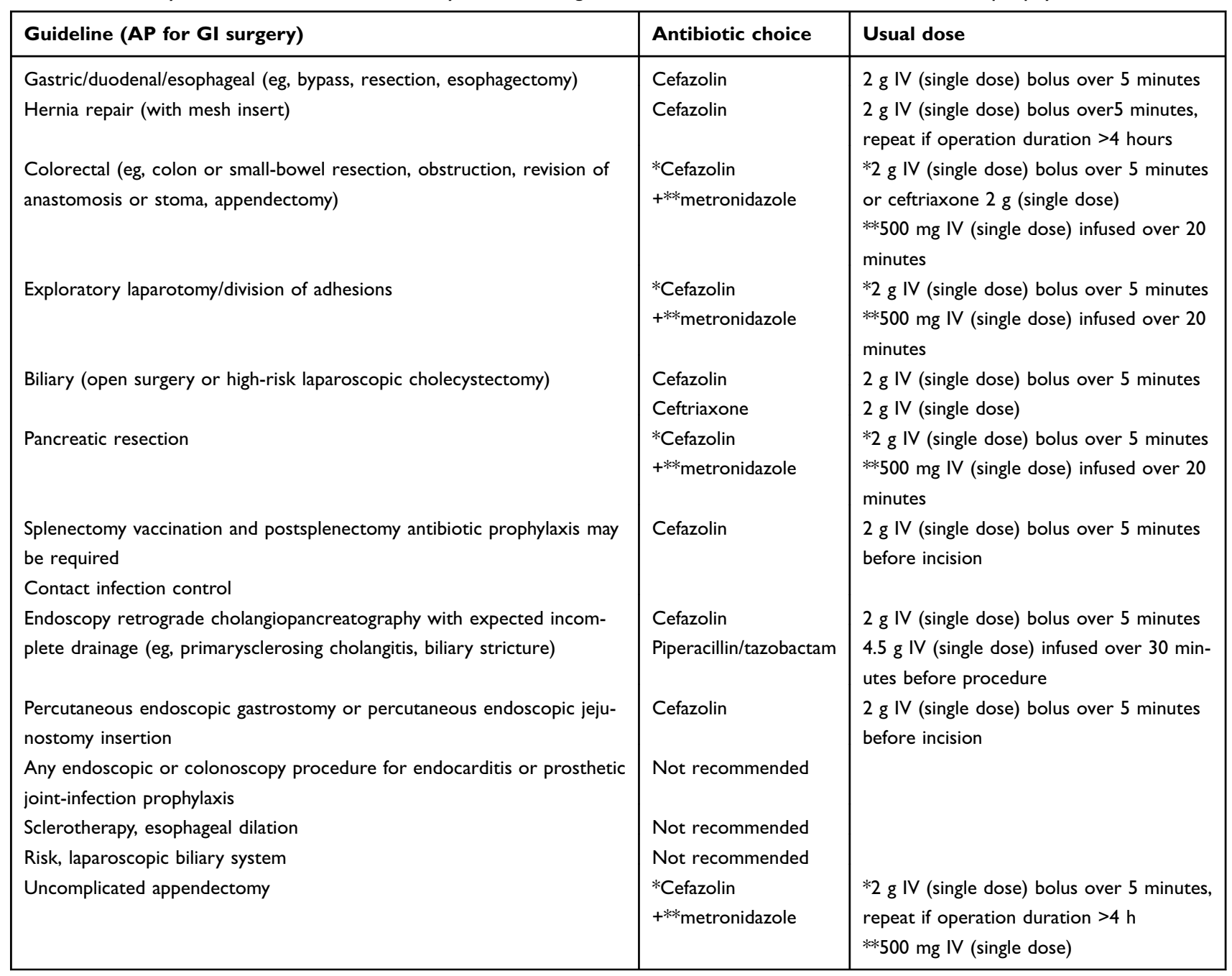

Notes: At the end of the intervention period, an infection-control specialist who was blinded to patient group recorded clinical data of patients in both groups. A Microsoft Excel table was designed to register type of surgical procedure, pharmacotherapy received, antibiotic usage (generic name, doses, dose intervals, and durations of administration), and costs (costs of hospitalization and antibiotics).

Abbreviations: AP, antimicrobial prophylaxis; GI, gastrointestinal; IV, intravenous.

Before the intervention period, a project consisting of revising the institutional guidelines for surgical antimicrobial prophylaxis based on the aforementioned guidelines, assigning a clinical pharmacist to the surgical department and arranging several meetings with the surgical department on the appropriate utilization of antibiotics, was developed in February 2018. Senior clinical pharmacists trained in infectious diseases would deliver lectures about SAP to key members of surgical departments.

Interventions consist of participating in ward rounds, attending recovery rooms, and making drug-treatment plans, and communicating immediately with surgeons in cases of discordance with guidelines. Educational sessions and handouts containing an abstract and general tips on SAP guidelines about AP for medical teams, especially surgical residents, were provided.
In each stage, data on the characteristics of the surgical patients were collected and the rationality of SAP during the perioperative period was evaluated according to the established criteria (Table 1). Data on irrational SAP were collected and evaluated by clinical pharmacists. In the pre intervention phase, discordance with the guidelines was recorded, but in the second phase (postintervention) clinical pharmacists communicated with the doctors responsible for order entry directly for correction of unreasonable cases and advice on rationality of the prescription.

Medical records of included patients, such as surgery characteristics and SAP (agent type, doses, dose intervals, number of doses, administration duration, and cost) were reviewed and recorded. SSIs were defined by the US Centers for Disease Control and 
Prevention definitions for surgical site-infection surveillance. ${ }^{11}$ At the end of the intervention period, an infection-control nurse who was blinded to patient group recorded the clinical data of patients in both groups.

\section{Identification of SSI cases}

Early SSI diagnosis was performed using clinical observation by surgeons. In the laboratory, different blood parameters, such as white blood-cell count, erythrocytesedimentationrate, and CRP, were assessed in patients suspected of infection. Then, patient samples, such as blood and wound-discharge samples, were evaluated using various microbial-culture techniques to confirm SSIs.

\section{Statistical analysis}

Data were analyzed using SPSS 19.0 (SPSS). Comparisons between the groups were made on patient characteristics, incidence and rate of infection, choice of prophylaxis antibiotics, duration of prophylaxis-antibiotic use, length of hospitalization, and financial costs. Analyses were conducted using Student's $t$-test for continuous variables. As some continuous variables for data were not normally distributed, Mann-Whitney $U$ tests were used to evaluate the statistical significance of between-group differences. Rates were analyzed by comparison of proportions with Pearson $\chi^{2} . P \leq 0.05$ was considered statistically significant.

\section{Results}

\section{Data assessment}

We recorded 875 patients who were admitted to the gastrointestinal surgery department during the 8 months of the study period. Patient characteristics are shown in Table 2. Both groups were similar with respect to demographics and clinical characteristics (Table 1). The most frequently used prophylaxis antibiotic in the pre intervention group and postintervention group was cefazolin sodium and metronidazole, respectively. The distribution of antibiotics used in our study is shown in Table 3. There was a significant decrease in the proportion of patients who underwent inappropriate AP in the intervention phase $(91.40 \%)$ compared with the baseline phase $(30.11 \%$; $P<0.001$; Table 2). Likewise, there was a significant increase in the proportion of patients receiving AP for no more than 48 hours $(P<0.01)$. The most frequently reported type of discordance with the guidelines in the pre intervention group was longer duration of antibiotic usage than guideline recommendations $(P<0.001)$.

\section{Clinical and economic outcomes}

To evaluate clinical impact, we assessed influences of intervention on the length of hospital stay in both groups. There was a significant decrease in length of hospital stay in the postintervention group (4.33 days) compared with the pre intervention group (5.14 days). A significant

Table 2 Patient characteristics

\begin{tabular}{|l|l|l|l|l|}
\hline \multicolumn{2}{|l|}{} & Group I* & Group 2** & P-value \\
\hline \multicolumn{2}{|l|}{ Age (years) mean (SD) } & Female & $51.06(12.60 \%)$ & $51.58(13.14 \%)$ \\
\hline \multirow{2}{*}{$\begin{array}{l}\text { Sex, } \\
\text { n (\%) }\end{array}$} & Male & $207(46.52 \%)$ & $234(54.42 \%)$ \\
\hline \multirow{2}{*}{$\begin{array}{l}\text { Surgical type, } \\
\text { n (\%) }\end{array}$} & Biliary & $238(53.48 \%)$ & $196(45.58 \%)$ \\
\cline { 2 - 5 } & Colorectal & $38(8.54 \%)$ & $6(1.40 \%)$ & 0.232 \\
\cline { 2 - 5 } & Exploratory laparotomy & $318(71.46 \%)$ & $338(78.60 \%)$ \\
\cline { 2 - 5 } & Gastrectomy & $38(8.54 \%)$ & $43(10.00 \%)$ \\
\cline { 2 - 5 } & Hernia repair & $19(4.27 \%)$ & $18(4.19 \%)$ \\
\cline { 2 - 5 } & Splenectomy & $25(5.62 \%)$ & $21(4.88 \%)$ \\
\hline \multirow{5}{*}{ Serum creatinine (SD) } & $7(1.57 \%)$ & $4(0.93 \%)$ \\
\hline
\end{tabular}

Notes: *Before Intervention; **after intervention. 
Table 3 Rationality of prophylactic-antibiotic selection

\begin{tabular}{|c|c|c|c|c|}
\hline & & Group I*, n (\%) & Group2**, n (\%) & $P$-value \\
\hline \multirow[t]{2}{*}{ Timing } & $0-48$ hours & 35 (7.87\%) & 405 (94.19\%) & \multirow[t]{2}{*}{$<0.001$} \\
\hline & $>48$ hours & $12 \mid(92.14 \%)$ & $25(5.71 \%)$ & \\
\hline \multirow[t]{10}{*}{ Selection } & Ceftriaxone & 156 (35.06\%) & 74 (17.21\%) & \multirow[t]{10}{*}{0} \\
\hline & Ciprofloxacin & $3(0.67 \%)$ & 0 & \\
\hline & Metronidazole & $203(45.62 \%)$ & $92(21.40 \%)$ & \\
\hline & Cefazolin & $16(3.60 \%)$ & $264(61.39 \%)$ & \\
\hline & Vancomycin & $19(4.27 \%)$ & 0 & \\
\hline & Imipenem & 27 (6.07\%) & 0 & \\
\hline & Clindamycin & $3(0.67 \%)$ & 0 & \\
\hline & Meropenem & $3(0.67 \%)$ & 0 & \\
\hline & Ampibactam & $12(2.70 \%)$ & 0 & \\
\hline & Teicoplanin & $3(0.67 \%)$ & 0 & \\
\hline \multicolumn{2}{|c|}{ Appropriateness } & 134 (30.1 I\%) & 393 (9l.40\%) & $<0.001$ \\
\hline
\end{tabular}

Notes: *Preintervention group; **postintervention group.

decrease was also noted in the SSI rate in the intervention group compared to the baseline group $(P=0.01)$. The mean cost of AP for patients in the baseline and intervention groups was US\$647 and $\$ 56$, respectively $(P<0.001)$ between two groups. In the pre intervention group, the mean cost of the antibiotic prescriptions was 11.5 times greater than the case group (Table 4). There were no changes in the prices of antibiotics during the two phases.

\section{Discussion}

Wth the aim of reducing the incidence of SSI, SAP is an adjunct to surgical procedures. To our knowledge, this is the first interventional study for gastrointestinal surgery focusing on clinical and economical impact of IDSAguideline implementation in Iran. In this study, guideline implementation by clinical pharmacist intervention led to a significant improvement in prophylactic-antibiotic prescription, with favorable economic and clinical outcomes.
These favorable results were achieved via correction of antibiotic choices, their timing of preoperative administration, and proper duration of AP, while unnecessary prescriptions and inappropriate combinations were decreased. Also, our intervention resulted in shortened length of hospital stay and decreased medication cost and SSI rate. This study revealed poor adherence to the prophylaxis guidelines in the pre intervention phase. This lack of adherence was attributed mainly to the absence of comprehensive institutional guidelines, easy accessibility to several antibiotics that were not included in the guidelines, and the preference of surgeons to overuse antimicrobial prophylactic drugs to decrease the risk of infection. Similar findings have been reported by other studies. ${ }^{15-17}$

During the intervention phase, patients received optimized antibiotic prescriptions, with a significant mean cost reduction of \$591. A major reason for this cost reduction was a significant decrease in the duration of antibiotic use

Table 4 Clinical and economic outcomes before and after intervention

\begin{tabular}{|l|l|l|l|}
\hline & Pre intervention & Post intervention & P-value \\
\hline Mean prophylactic-antibiotic cost (US\$), mean (SD) & $647(20.80)$ & $56(\mathrm{I} .80)$ & $<0.00 \mathrm{I}$ \\
Length of hospitalization, days, (SD) & $5.14(2.42)$ & $4.33(\mathrm{I} .04)$ & $<0.00 \mathrm{I}$ \\
Rate of surgical site infection, n (\%) & $30(6.74)$ & $13(3.02)$ & 0.01 \\
\hline
\end{tabular}


in the intervention group (94.19\%) compared to the baseline group $(7.87 \% ; P<0.001)$. In Iran, the government provides most of the financial resources needed for the health-care system. Despite the fact that most generic drugs, including antibiotics, are produced in Iran, the extended use of these drugs imposes a huge financial burden on the Ministry of Health. ${ }^{18}$ Therefore, adherence to prophylaxis guidelines can avoid any unnecessary costs in this context.

Research has revealed that prolongation of AP after surgery is not necessary. In fact, the extended use of antibiotics increases the risk of antibiotic resistance and imposes a financial burden on patients and hospitals. ${ }^{19}$ However, prolongation of AP, as was seen in the pre intervention group of this study, continues to be the main point of noncompliance with guidelines in surgery wards. Several published guidelines suggest that a single dose of cefazolin is sufficient for preventing SSIs in most gastrointestinal surgeries. ${ }^{20} \mathrm{~A}$ meta-analysis performed by Shen et $\mathrm{al}^{21}$ did not find any additional advantage for postoperative maintenance antibiotics. Another study has shown that prolonged antibiotic use increases the risk of resistant strains without any advantage. ${ }^{22}$ Similarly, in our report, prolongation of antibiotic administration was not associated with any decrease in SSI rate in the pre intervention group, and interestingly this rate was higher than the post intervention group. Emerging drug-resistant strains due to prolonged antibiotic usage are a possible reason for this phenomenon. Another reason could be improvement in knowledge about infection control when the surgery team are involved in teaching sessions about SAP and they are aware that their practice is observed by another authority. However, more studies are needed to clarify how adherence to the guidelines diminishes the SSI rate compared to antibiotic overuse.

Ideally, a guideline for surgical prophylaxis should stop SSIs, stop SSI-related morbidity and mortality, reduce the duration of health care and related financial cost, ${ }^{23,24}$ and generate no adverse effects. ${ }^{25}$ Over the past few decades, several organizations, hospitals, and government agencies, including the IDSA, have established national qualityimprovement initiatives to further improve the safety and outcomes of surgery. ${ }^{14}$ One aspect of focus in these initiatives for patients undergoing surgery is the prevention of SSIs. According to studies in US hospitals, application of updated international guidelines, such as those of the IDSA, is an efficient, sensitive, and reliable method of surveillance of antimicrobial prophylaxis. ${ }^{26}$ At the same time, similar guidelines have been revealed to be of value and are now established in several hospitals in some European countries. ${ }^{27}$

The implementation of institutional guidelines for SSI prophylaxis plays a crucial role in guiding surgeons to use the most appropriate antibiotics and doses based on evidence-based recommendations. These guidelines should be tailored to the needs of each institution, based on the pattern of antimicrobial resistance, most common types of surgeries performed, and the antibiotics available. In 2004, a multicenter prospective Japanese study assessed the efficacy of a protocol of prophylactic-antibiotic use to prevent SSIs in urological surgery. The results of this study showed that using protocols efficiently decreased the amount of antibiotics used and consequently financial cost without increasing infection rates. ${ }^{28}$ Another study conducted by Trinchieri et $\mathrm{al}^{29}$ was designed to evaluate incidence of SSIs in a urology ward after application of the European Association of Urology guidelines. They concluded that antimicrobial prophylaxis according to the association's guidelines seemed to be adequate to prevent symptomatic/febrile genitourinary infections. Additional cost savings might be realized through collaborative management by surgeons and clinical pharmacists to choose the most cost-effective drug and reduce or eliminate postoperative dosing. ${ }^{30,31}$ In addition, the application of standardized antibiotic order sets, automatic stop-order programs, and educational courses has been also shown to facilitate the adoption of updated guidelines for surgical antimicrobial prophylaxis. ${ }^{32-36}$

A significant improvement in adherence to antimicrobial-prophylaxis guidelines was noted in the current study after implementation of a quality-improvement project. The provision of educational sessions and assignment of a clinical pharmacist in a surgery department clearly played a major role in the improved compliance. In the current study, the authors believe that the presence of a clinical pharmacist in a surgical department was essential in encouraging adherence to the revised antimicrobial-prophylaxis guidelines. This clinical pharmacist helped with specific recommendations related to prescribing antibiotics for surgical residents. It is necessary to implement local guidelines and continuous audit and feedback to surgeons regarding the appropriateness of their practice to achieve sustained clinical and economic improvement in prophylactic-antibiotic usage.

Several limitations of this study should be acknowledged. First of all, it had pre- vs post intervention design. 
This prospective study was less convincing than a randomized controlled trial. Second, our study was done in 8 months. We did not evaluate the sustained effects of guideline implementation after the interventions had finished. Future studies are recommended to explore duration of adherence to antimicrobial-prophylaxis guidelines.

\section{Conclusion}

In the present study, IDSA-guideline implementation through a quality-improvement project significantly improved the rational use of AP among gastrointestinal surgeons and residents and reduced antibiotic-usage costs and length of hospitalization. It is essential for surgeons to be aware of the importance of adherence to AP guidelines and to know that this adherence has a positive impact on patient outcomes. The results of our study emphasize that clinical pharmacists can help to enhance adherence to institutional antimicrobial-prophylaxis guidelines and decrease irrational drug utilization.

\section{Acknowledgment}

This research was supported by Shiraz University of Medical Sciences (94-01-05-10183).

\section{Disclosure}

The authors report no conflicts of interest in this work.

\section{References}

1. Roth JA, Juchler F, Dangel M, Eckstein FS, Battegay M, Widmer AF. Frequent door openings during cardiac surgery are associated with increased risk for surgical site infection: a prospective observational study. Clin Infect Dis. 2018;69:290-294.

2. Organization, WH. Global Guidelines for the Prevention of Surgical Site Infection; 2018.

3. Puoti M, Greco DP, Merli M, Abbati C. Post-surgical hidden costs: infections. In: Abdominal Wall Surgery. Heidelberg: Springer; 2019:127-139.

4. Gillespie BM, Bull C, Walker R, Lin F, Roberts S, Chaboyer W. Quality appraisal of clinical guidelines for surgical site infection prevention: a systematic review. PLoS One. 2018;13(9):e0203354.

5. GlobalSurg Collaborative. Surgical site infection after gastrointestinal surgery in high-income, middle-income, and low-income countries: a prospective, international, multicentre cohort study. Lancet Infect Dis. 2018;18(5):516-525.

6. Eshrati B, Masoumi Asl H, Afhami S, Pezeshki Z, Seifi A. Health careassociated infections in Iran: a national update for the year 2015. Am J Infect Control. 2018;46(6):663-667. doi:10.1016/j.ajic.2017.11.017

7. Khazaei S, Khazaei S, Ayubi EJ. Importance of prevention and control of nosocomial infections in Iran. Iran J Public Health. 2018;47 (2):307-308.

8. Sommerstein R, Atkinson A, Kuster SP, et al. Antimicrobial Prophylaxis and the Prevention of Surgical Site Infection in Cardiac Surgery: an Analysis of 21007 Patients in Switzerland. Eur J Cardiothorac Surg. 2019.
9. Ierano C, Peel T, Ayton D, et al. Surgical antibiotic prophylaxis-the evidence and understanding its impact on consensus guidelines. Infect Dis Health. 2018;23(3):179-188.

10. Kwak YG. Appropriate use of surgical antibiotic prophylaxis. $J$ Korean Med Sci. 2019;34(17):e136.

11. Sway A, Solomkin JS, Pittet D, Kilpatrick C. Methodology and background for the World Health Organization global guidelines on the prevention of surgical site infection. Surg Infect (Larchmt). 2018;19(1):33-39. doi:10.1089/sur.2017.076

12. Satti M, Hamza M, Sajid Z, et al Compliance Rate of Surgical Antimicrobial Prophylaxis and Its Association with Knowledge of Guidelines among Surgical Residents in a Tertiary Care Public Hospital of a Developing Country. 2019.

13. Ariyo $\mathrm{P}$, et al. Implementation strategies to reduce surgical site infections: a systematic review. Infect Control Hosp Epidemiol. 2019;40:1-14.

14. Bratzler DW, Dellinger EP, Olsen KM, et al. Clinical practice guidelines for antimicrobial prophylaxis in surgery. Surg Infect (Larchmt). 2013;14(1):73-156. doi:10.1089/sur.2013.9999

15. Purba AKR, Setiawan D, Bathoorn E, Postma MJ, Dik JH, Friedrich AW. Prevention of surgical site infections: a systematic review of cost analyses in the use of prophylactic antibiotics. Front Pharmacol. 2018;9:776.

16. Mujagic E, Marti WR, Coslovsky M, et al. Associations of Hospital Length of Stay with Surgical Site Infections. World J Surg. 2018;42 (12):3888-3896. doi:10.1007/s00268-018-4733-4

17. Goldberg H, Shenhar C, Tamir H, et al. Predictors of surgical site infection after radical cystectomy: should we enhance surgical antibiotic prophylaxis?. World J Urol. 2018;37:1-7.

18. Cheraghali AM. Trends in Iran pharmaceutical market. Iran $J$ Pharm Res. 2017;16(1):1.

19. Hawn MT, Knowlton LM. Balancing the risks and benefits of surgical prophylaxis: timing and duration do matter. JAMA Surg. 2019.

20. Voit SB, Todd JK, Nelson B, Nyquist A-C. Electronic surveillance system for monitoring surgical antimicrobial prophylaxis. Pediatrics. 2005;116(6):1317-1322. doi:10.1542/peds.2004-1969

21. Shen J, Sun Q, Zhou X, et al. Pharmacist interventions on antibiotic use in inpatients with respiratory tract infections in a Chinese hospital. Int $J$ Clin Pharm. 2011;33(6):929-933. doi:10.1007/s11096-011-9577-z

22. Harbarth S, Samore MH, Lichtenberg D, Carmeli Y. Prolonged antibiotic prophylaxis after cardiovascular surgery and its effect on surgical site infections and antimicrobial resistance. Circulation. 2000;101(25):2916-2921. doi:10.1161/01.cir.101.25.2916

23. Threapleton CJ, Janjua S, Fortescue R, Baker EH. Head-to-head oral prophylactic antibiotic therapy for chronic obstructive pulmonary disease. Cochrane Database Syst Rev. 2019;5:CD013024.

24. Laituri C, Arnold MA. A standardized guideline for antibiotic prophylaxis in surgical neonates. in Seminars in pediatric surgery. Semin Pediatr Surg. 2019;28:59-56. Elsevier.

25. Kanayama M, Hashimoto T, Shigenobu K, Oha F, Togawa D. Effective prevention of surgical site infection using a Centers for Disease Control and Prevention guideline-based antimicrobial prophylaxis in lumbar spine surgery. J Neurosurg Spine. 2007;6(4):327329. doi:10.3171/spi.2007.6.4.7

26. Pestotnik SL, Classen DC, Evans RS, Burke JP. Implementing antibiotic practice guidelines through computer-assisted decision support: clinical and financial outcomes. Ann Intern Med. 1996;124(10):884890. doi:10.7326/0003-4819-124-10-199605150-00004

27. Dunn K, O’Reilly A, Silke B, Rogers T, Bergin C. Implementing a pharmacist-led sequential antimicrobial therapy strategy: a controlled before-and-after study. Int J Clin Pharm. 2011;33(2):208-214. doi:10.1007/s11096-010-9475-9

28. Yamamoto S, Kanamaru S, Kunishima Y, Ichiyama S, Ogawa O. Perioperative antimicrobial prophylaxis in urology: a multi-center prospective study. J Chemother. 2005;17(2):189-197. doi:10.1179/ joc.2005.17.2.189 
29. Trinchieri A, Paparella S,Cappoli S, et al. Prospective assessment of the efficacy of the EAU guidelines for the prevention of nosocomial acquired infections after genitourinary surgery in a district hospital. Arch Ital Urol Androl. 2009;81(1):46-50.

30. Gómez MI, Acosta-Gnass SI, Mosqueda-Barboza L, Basualdo JA. Reduction in surgical antibiotic prophylaxis expenditure and the rate of surgical site infection by means of a protocol that controls the use of prophylaxis. Infect Control Hosp Epidemiol. 2006;27(12):13581365. doi: $10.1086 / 509845$

31. Bond C, Raehl CL. Clinical and economic outcomes of pharmacistmanaged antimicrobial prophylaxis in surgical patients. AmJ Health Syst Pharm. 2007;64(18):1935-1942. doi:10.2146/ajhp060631

32. Hermsen ED, Smith Shull S, Puumala SE, Rupp ME. Improvement in prescribing habits and economic outcomes associated with the introduction of a standardized approach for surgical antimicrobial prophylaxis. Infect Control Hosp Epidemiol. 2008;29(5):457-461. doi:10. $1086 / 587811$
33. Alerany C, Campany D, Monterde J, Semeraro C. Impact of local guidelines and an integrated dispensing system on antibiotic prophylaxis quality in a surgical centre. $J$ Hosp Infect. 2005;60(2):111-117. doi:10.1016/j.jhin.2004.07.022

34. Kritchevsky SB, Braun BI, Bush AJ, et al. The effect of a quality improvement collaborative to improve antimicrobial prophylaxis in surgical patients: a randomized trial. Ann Intern Med. 2008;149:472480. doi:10.7326/0003-4819-149-7-200810070-00007

35. Potenza B, Deligenica M, Estigoy B, et al. Lessons learned from the institution of the Surgical Care Improvement Project at a teaching medical center. Am J Surg. 2009;198:881-888. doi:10.1016/j.amjsurg.2009.08.015

36. Manniën J, Van Kasteren M, Nagelkerke NJ, et al. Effect of optimized antibiotic prophylaxis on the incidence of surgical site infection. Infect Control Hosp Epidemiol. 2006;27:1340-1346. doi:10.1086/509842

\section{Publish your work in this journal}

Infection and Drug Resistance is an international, peer-reviewed openaccess journal that focuses on the optimal treatment of infection (bacterial, fungal and viral) and the development and institution of preventive strategies to minimize the development and spread of resistance. The journal is specifically concerned with the epidemiology of antibiotic resistance and the mechanisms of resistance development and diffusion in both hospitals and the community. The manuscript management system is completely online and includes a very quick and fair peerreview system, which is all easy to use. Visit http://www.dovepress.com/ testimonials.php to read real quotes from published authors. 